\title{
Semaphorin 3B Gene Suppresses Tumor Growth Through the p53 Signaling Pathway and Neuropilin Receptors
}

\author{
Yan Ma, Mingming Fang, Xifa Zhou* \\ Department of Radiation Oncology, Changzhou Tumor Hospital, Soochow University, Changzhou, China \\ Email address: \\ 1660487936@qq.com (Yan Ma),vsword@163.com (Mingming Fang),251860135@qq.com (Xifa Zhou) \\ ${ }^{*}$ Corresponding author
}

\section{To cite this article:}

Yan Ma, Mingming Fang, Xifa Zhou. Semaphorin 3B Gene Suppresses Tumor Growth Through the p53 Signaling Pathway and Neuropilin Receptors. American Journal of Clinical and Experimental Medicine. Vol. 5, No. 6, 2017, pp. 234-238. doi: 10.11648/j.ajcem.20170506.18

Received: October 28, 2017; Accepted: November 9, 2017; Published: December 29, 2017

\begin{abstract}
The semaphorin family has been demonstrated to possess tumor suppressor activity; semaphorin 3B (SEMA3B) is differentially expressed in several types of tumors, and has been identified as a tumor suppressor gene. SEMA3B is shown to be a target gene of $\mathrm{p} 53$, and it suppresses tumor growth through the p53 signaling pathway. The mechanisms underlying tumor suppression by SEMA3B include neuropilin receptors (NRP1 and NRP2), which reduce the action of vascular endothelial growth factor (VEGF), thus, inhibiting tumor angiogenesis. Deficiency or down-regulation of SEMA3B expression can be found in a variety of malignant tumors including lung cancer, ovarian cancer, nervous system tumors, and hepatobiliary tumors, and this suppression involves methylation, loss of heterozygosity $(\mathrm{LOH})$ and enzyme cleavage. This review summarizes recent research approaches on the tumor suppression effects and mechanisms of SEMA3B.
\end{abstract}

Keywords: Semaphorin 3B, p53, Malignant Tumor, Tumor Suppression, Mechanism

\section{Introduction}

The semaphorins area large protein superfamily that is mainly composed of membrane proteins or secreted proteins $[1,2]$. Semaphorin 3B (SEMA3B), a tumor suppressor gene, is a member of the semaphorin family and is located on $3 \mathrm{p} 21.3$ [3]. Research shows that the SEMA3B protein may play a role in regulating cell growth, and it has been identified as a mediator of the p53 tumor-suppressor $[4,5]$. The mechanisms underlying the tumor suppression by SEMA3B include a combination of neuropilins receptors (NRP1 and NRP2) that reduce the action of vascular endothelial growth factor (VEGF) thus inhibiting tumor angiogenesis $[6,7]$. SEMA3B is inactivated in lung cancer [8, 9], HEY ovarian carcinoma [10], hepatocellular carcinomas and cholangiocarcinomas [11]; activation involves methylation, loss of heterozygosity (LOH) and enzyme cleavage. However, SEMA3B also exerts unexpected functions in cancer progression by fostering a pro-metastatic environment [12]. This review summarizes recent research on the tumor suppression effects and mechanisms of SEMA3B.

\section{The Semaphorin Family and SEMA3B}

The SEMA3B gene is a member of the semaphorin gene family, which was found in the developing locust nervous system by Kolodkin et al. in 1992 [13]. The semaphorin family is divided into 8 subgroups, of which subgroups 3-7 are present in vertebrates [14]. The semaphorin family has a common Sema domain, which plays a very important role in semaphorin signaling transduction and receptor specific binding [15]. Plexin and Met/Ron receptors also have Sema domains and, together with the semaphorion family, are referred to as part of the semaphorin superfamily [16]. The sema domain is involved in the regulation of NRP pathway-dependent apoptosis.

Recent studies have indicated that semaphorin plays an important role in nervous system development, immune responses, angiogenesis and tumor development $[1,15]$. The semaphorin gene encodes three types of proteins including transmembrane proteins, membrane proteins and secreted proteins. The third subgroup of semaphorins has been shown to includea tumor suppressor gene, which is located in the 21.3 region of the short arm of chromosome 3 and encodes a 
secreted proteins [10].

\section{Tumor Suppression of SEMA3B}

SEMA3B is regarded as a target gene of p53, a very important tumor suppressor gene with a high mutation rate $[8$, 17]. SEMA3B may play a vital role in inhibiting tumors through the p53 signaling pathway. Electrophoretic mobility shift assays (EMSA) confirmed that the promoter region of SEMA3B contains a p53 protein binding site (P53BS), and the regulation of the transcriptional expression of SEMA3B is p53 dependent. By using restriction enzymes and recombination technology, Moriya et al. [18] built a pcDNA-SEMA3B eukaryotic expression vector and introduced the SEMA3B gene into a non-small cell lung cancer cell line, NCI-H1299. The authors found that the cells over-expressing SEMA3B had less clone formation compared to the control group $(\mathrm{P}<0.01)$, suggesting that SEMA3B induced apoptosis in tumor cells. Ochi et al. [3] reported that the introduction of exogenoushp53 into a glioblastoma cell line lacking wild-type p53 (U373MG) dramatically induced the expression of SEMA3B mRNA. Further studies demonstrated that a p53 binding site exists in the SEMA3B promoter region; shares $90 \%$ identity with the p53 binding sequence, which suggests that SEMA3B is a target gene of wild-type p53. SEMA3B mRNA expression in the p53 wild-type breast cancer cell line MCF-7 increased and reached its highest value 72 hours after treatment with ultraviolet (UV) irradiation or adriamycin, both of which can cause DNA damage in the cells [3]. The result was consistent with that of direct p53 gene interference. However, SEMA3B mRNA expression in p53-negative H1299 lung cancer cells was unchanged under the same conditions, suggesting that up-regulation of SEMA3B expression by UV irradiation or adriamycin is p53 dependent. Renpin et al. [19] found that increase in SEMA3B mRNA expression in the gastric cancer cell lines $\mathrm{BGC}-823$ and MGC-803 can up-regulate the expression of p53 mRNA. However, by introducing exogenous SEMA3B into the lung cancer cell line H1299, which lacksthe wild-type p53 gene, Rivera et al. [7] found that the cells that over-expressed SEMA3B had a reduced number of colonies in colony formation assays compared to the control group $(\mathrm{P}<0.01)$. This suggests that SEMA3B can inhibit growth of tumor cells independent of p53. The study by Ito et al. [20] did not find a relationship between p53 and SEMA3B methylation in non-small cell lung cancer.

VEGF is a very important factor that promotes the growth of blood vessels. Both the SEMA3 family and VEGF have the same transmembrane receptors neuropilin-1 (NP-1) and neuropilin-2 (NP-2) [21]. It has been shown that SEMA3B protein competitively binds with NP-1 and inhibits VEGFl65-induced tumor growth by inhibiting tumor angiogenesis and reducing the microvessel density (MVD) [22]. A study also found that the expression of SEMA3B mRNA in lung cancer tissue was negatively correlated with the MVD and the expression of VEGF mRNA [23].
In many malignant tumors, loss of heterozygosity ( $\mathrm{LOH}$ ) is very common in the $3 \mathrm{p} 21.3$ region, suggesting that the region may contain a tumor suppressor gene. The SEMA3B gene, which is located in $3 \mathrm{p} 21.3$, has shown different degrees of deficiency or down-regulation in many tumors. Tomizawa et al. [24] transfected exogenous SEMA3B genes into lung cancer NCI-H1299 cells lacking expression of SEMA3B and SEMA3F and found that the transfected H1299 cells formed $90 \%$ fewer cell colonies compared to the control group. The cells transfected with mutation SEMA3Bhaving a single amino acid missense mutation formed $30 \%-40 \%$ fewer cell colonies compared to the control group. These mutation sites, including codons $328,397,415$ and 561, are located in the SEMA3B domain. In addition, a H460-M cell line derived from the lung cancer cell line NIH-H460 exhibited enhanced spontaneous metastasis in nude mice. Lange et al. [25] reported that the SEMA3B mRNA expression in H460-M cell was significantly decreased compared to NIH-H460 cells. Recent research has shown that the expression of SEMA3B in prostate cancer and ovarian cancer was down-regulated by different degrees [5, 26]. Tse et al. [10] found that the expression of SEMA3B in the HEY ovarian cancer cell line decreased by approximately 25-folds compared to normal ovarian tissues. By transfecting HEY cells with an exogenous SEMA3B gene and injecting the cells into Balb/c nude mice, they found that injection of transfected HEY for 38 days inhibited tumor growth compared to non-transfected HEY injection (with tumor volumes of 8.0-740 $\mathrm{mm}$ vs $933 \mathrm{~mm}$, respectively). The in vitro study demonstrated that the SEMA3B-transfected HEY cells incubated with soft agar culture showed nearly 10 times less colony formation compared to non-transfected cells. In addition, transfection of exogenous SEMA3B combined with paclitaxel or serum starvation showed synergistic effects on tumor inhibition, indicating that the SEMA3B gene can enhance the sensitivity of HEY cells to the toxicity of paclitaxel or serum starvation. To analyze the relationship between the levels of SEMA3B expression and average overall survival periods, Karayan et al. [27] divided 38 patients with neuroglioma into three groups based on the expression levels of SEMA3B in samples of their tumors. The results showed that patients with SEMA3B expression of less than $25 \%$, between $25 \%$ and $75 \%$ and greater than $75 \%$ had average overall survival periods of 9,17 and 24 months, respectively, suggesting that SEMA3B can prolong survival-period. Tischoff et al. [11] found that the mRNA expression of SEMA3B was down-regulated in hepatocellular carcinomas, gallbladder cancer and cholangiocarcinomas independent of the stage of the tumors. The authors also analyzed the expression of SEMA3B in 35 cases of hepatocellular carcinoma and 15 cases of cholangiocarcinomas and found that 29 cases of hepatocellular carcinomas and all cholangiocarcinomas cases showed both the absence or down-regulation of SEMA3B and methylation in the promoter region of SEMA3B. The mRNA expression of SEMA3B in 60 gastric cancer cases was significantly lower than that in normal gastric mucosa. 


\section{Inactivation of SEMA3B Tumor Suppression and the Relationship to Methylation, LOH and Enzyme Cleavage}

Inactivation of SEMA3B tumor suppression is closely related to its methylation, $\mathrm{LOH}$ and enzyme cleavage. Methylation is the primary factor contributing to the inactivation of human tumor suppressor genes [28-31]. Kuroki et al. [32] analyzed the methylation status of the SEMA3B promoter and $\mathrm{LOH}$ at 3 p21.3 in eight non-small cell lung cancer cell lines and 27 primary tumors. The results showed that hypermethylation of SEMA3B was found in $50 \%$ of the non-small cell lung cancer cell lines and $41 \%$ of the primary tumors studied. Notably, 7 of the 8 tumors with both hypermethylation and LOH of SEMA3B showed the absence of the SEMA3B expression. Tomizawa et al. [24] determined the methylation of 4 types of lung cancer cell lines with an absence of SEMA3B expression and 2 types of lung cancer cell lines with the presence of SEMA3B expression. It was observed that the former has a dense $\mathrm{CpG}$ methylation island in the 5 ' region of the SEMA3B, while the later has no methylation of SEMA3B. Nair et al. [33] reported that $95 \%$ of neuroblastomas without the $3 p$ gene have methylation of $\mathrm{CpG}$ sites in the SEMA3B promoter; however, only $52 \%$ of neuroblastomas with the $3 p$ gene have methylation at the same site, suggesting the contribution of allelic inactivation to its regulation. Tischoff et al. [11] studied the methylation of the SEMA3B promoter in 35 cases with hepatocellular carcinomas and 15 cases with cholangiocarcinomas and found that $83 \%$ of the former cases and $100 \%$ of the later cases showed methylation of the SEMA3B promoter. They also found that methylation is independent of patient age and tumor grade or stage, suggesting that rmethylation of the SEMA3B promoter is a relatively early event. Numerous studies have indicated that the gene expression of SEMA3B can be induced by de-methylation agents [32, 33]. Kuroki et al. [32] reported that treatment with 5-AZAC, a de-methylation agent, restored SEMA3B expression in non small cell lung cancer cells. Similarly, Tischoff et al. [11] reported that HepG2, which lacks expression of SEMA3B, re-expressed SEMA3B after treatment with 5-AZAC. Nair et al. [33] found that SEMA3B expression in neuroblastoma cells increased significantly after treatment with another de-methylation agent, 5-aza-2-deoxycytidylate. However, Yi et al. [34] found that $100 \%$ of nasopharyngeal carcinoma samples and $73.3 \%$ of chronic nasopharyngitis tissue tested showed hypermethylation of the SEMA3B promoter, which suggests that hypermethylation of the SEMA3B promoter is related to inflammation, aging or other physiological and pathological conditions; that are un elated to the pathogenesis and development of nasopharyngeal carcinoma. Studies by Nair et al. [33] also indicated that cells without methylation of the SEMA3B promoter have low expression levels of SEMA3B, suggesting that methylation is not necessary for the inactivation of SEMA3B.

LOH is also regarded as a mechanism of SEMA3B inactivation and is closely related to methylation. Riquelme et al. [35] reported a very high frequency of SEMA3B methylation in gallbladder cancer $(46 / 50,92 \%)$. The author detected LOH at 8 microsatellite loci in the $3 \mathrm{p} 21.3$ region of the 32 samples and found that 14 samples demonstrated methylation in at least one site located in SEMA3B. Twelve out of 14 (86\%) gallbladder cancers demonstrated both $3 \mathrm{p} 21.3$ LOH and methylation of SEMA3B.

Tischoff et al. [11] analyzed the methylation status of SEMA3B and LOH at 3p21.3 in 35 hepatocellular carcinomas and 15 cholangiocarcinomas. The results showed that SEMA3B hypermethylation was found in 29/35 hepatocellular carcinomas $(83 \%)$ and in all $(15 / 15)$ patients with cholangiocarcinomas. $\mathrm{LOH}$ at $3 \mathrm{p} 21.3$ occurred in $8 / 35$ $(23 \%)$ hepatocellular carcinomas and $3 / 15 \quad(20 \%)$ cholangiocarcinomas. Yi et al. [34] reported that the total LOH frequency of SEMA3B in patients with nasopharyngeal carcinomas was $45 \%$, and the expression level of the SEMA3B gene was negatively correlated with $\mathrm{LOH}$. However, Osada et al. [36] did not find a correlation between $\mathrm{LOH}$ and mRNA expression deficiency of SEMA3B in ovarian epithelial samples. Ito et al. [20] also found that $\mathrm{LOH}$ without hypermethylation of the SEMA3B gene can also occur in some cases of non-small cell lung cancer. This suggests that just one allelic loss might also be sufficient for the inactivation of SEMA3B for lung cancer development.

Enzyme cleavage is considered another mechanism for SEMA3B inactivation. All the class-3 semaphorins, including SEMA3B contain a conserved consensus pro-protein convertase (PPC) cleavage site that is cleaved into two fragments of $\sim 60$ and $\sim 20 \mathrm{kDa}$ when combined with furin-like PPC, which leads to the inactivation of SEMA3B. Varshavsky et al. [22] found that furin-like PPC can inactivate SEMA3B, causing it to lose its ability to inhibit angiogenesis. However, the action of furin-like PPC on SEMA3B can be inhibited by a furin-like PPC inhibitor. The authors generated a SEMA3B variant of human embryonic kidney (HEK) 293 cellswith a point mutation at the PPC cleavage site (sema3B-m), thereby conferring resistance to cleavage. In contrast to HEK293 cells expressing native SEMA3B, cells expressing SEMA3B-m are strongly resistant to bFGF mediated angiogenesis. Conditioned medium from SEMA3B-m expressing cells rapidly caused the disassembly of focal adhesions and a collapse of the actin cytoskeleton of endothelial cells. The conditioned medium also inhibited the VEGFinduced phosphorylation of extracellular signal-regulated kinase $1 / 2$, induced apoptosis of endothelial cells, and inhibited the formation of tubes from endothelial cells, consequently inhibiting angiogenesis and tumor growth.

In summary, SEMA3B is a tumor suppressor gene, and deficiency or down-regulation of SEMA3B has been found in a variety of malignant tumors. The mechanisms involve methylation, $\mathrm{LOH}$ and enzyme cleavage. 


\section{Conclusion}

SEMA3B, as a downstream gene of $\mathrm{p} 53$, is considered to be a tumor suppressor gene in the signal transduction pathway ofp53 gene, inducing tumor cell apoptosis and inhibiting the growth of tumors. However, a few studies have reported that SEMA3B inhibited tumor growth while unexpectedly increasing tumor metastasis [37]. Although SEMA3B was found to be deficient or down-regulated at different levels in lung cancer, ovarian cancer and other malignant tumors, the relationship between SEMA3B and the differentiation and staging of tumors is still controversial. Nevertheless, the tendency is that the expression of SEMA3B is lower or more strongly down-regulated in patients with highly malignant tumors. The mechanism of inactivation of SEMA3B include methylation, $\mathrm{LOH}$ and enzyme cleavage. The existence of a link between methylation and $\mathrm{LOH}$ remains controversial. In summary, the role of SEMA3B in the development of cancer is complex and in-depth studies can provide clues for discovering drug targets.

\section{Acknowledgements}

This work is supported by the Changzhou High-Level Medical Talents Training Project (2016C2BJ016; 2016C2LJ026), the Jiangsu Provincial Special Program of Medical Science (BE2015631), the Natural Science Foundation of Jiangsu (BK20151174), and the Changzhou Scientific Program (CJ20159038; CJ20140050; CE20155046; QN201503)

\section{References}

[1] Sang C, Zhang Y, Chen F, Huang P, Qi J, Wang P, Zhou Q, Kang $\mathrm{H}$, Cao $\mathrm{X}$ and Guo L: Tumor necrosis factor alpha suppresses osteogenic differentiation of MSCs by inhibiting semaphorin $3 \mathrm{~B}$ via $\mathrm{Wnt} /$ beta-catenin signaling in estrogen-deficiency induced osteoporosis. Bone 84: 78-87, 2016.

[2] Arbeille E, Reynaud F, Sanyas I, Bozon M, Kindbeiter K, Causeret F, Pierani A, Falk J, Moret F and Castellani V: Cerebrospinal fluid-derived Semaphorin 3B orients neuroepithelial cell divisions in the apicobasal axis. Nat Commun 6: 6366, 2015.

[3] Ochi K, Mori T, Toyama Y, Nakamura Y and Arakawa H: Identification of semaphoring $3 \mathrm{~B}$ as a direct target of $\mathrm{p} 53$. Neoplasia 4: 82-87, 2002.

[4] Wang H, Jiang L, Gao B and Dong M: Alteration of serum semaphorin 3B levels in preeclampsia. Clin Chim Acta 455: 60-63, 2016.

[5] Joseph D, Ho SM and Syed V: Hormonal regulation and distinct functions of semaphorin-3B and semaphorin-3F in ovarian cancer. Mol Cancer Ther 9: 499-509, 2010.

[6] Fonseca FP, Bingle L, Santos-Silva AR, Lopes MA, de Almeida OP, de Andrade BA, Mariano FV, Kowalski LP, Rangel AL, et al: Semaphorins and neuropilins expression in salivary gland tumors. J Oral Pathol Med 45: 119-126, 2016.
[7] Castro-Rivera E, Ran S, Thorpe P and Minna JD: Semaphorin 3B (SEMA3B) induces apoptosis in lung and breast cancer, whereas VEGF165 antagonizes this effect. Proc Natl Acad Sci U S A 101: 11432-11437, 2004.

[8] Smardova J, Liskova K, Ravcukova B, Malcikova J, Hausnerova J, Svitakova M, Hrabalkova R, Zlamalikova L, Stano-Kozubik K, et al: Complex analysis of the p53 tumor suppressor in lung carcinoma. Oncol Rep 35: 1859-1867, 2016.

[9] Loginov VI, Dmitriev AA, Senchenko VN, Pronina IV, Khodyrev DS, Kudryavtseva AV, Krasnov GS, Gerashchenko GV, Chashchina LI, et al: Tumor Suppressor Function of the SEMA3B Gene in Human Lung and Renal Cancers. PLoS One 10: $\mathrm{e} 0123369,2015$.

[10] Tse C, Xiang RH, Bracht T and Naylor SL: Human Semaphorin 3B (SEMA3B) located at chromosome 3p21.3 suppresses tumor formation in an adenocarcinoma cell line. Cancer Res 62: 542-546, 2002.

[11] Tischoff I, Markwarth A, Witzigmann H, Uhlmann D, Hauss J, Mir mohammad sadegh A, Wittekind C, Hengge UR and Tannapfel A: Allele loss and epigenetic inactivation of $3 \mathrm{p} 21.3$ in malignant liver tumors. Int J Cancer 115: 684-689, 2005.

[12] Lin ZF, Shen XY, Lu FZ, Ruan Z, Huang HL and Zhen J: Reveals new lung adenocarcinoma cancer genes based on gene expression. Eur Rev Med Pharmacol Sci 16: 1249-1256, 2012.

[13] Kolodkin AL, Matthes DJ and Goodman CS: The semaphorin genes encode a family of transmembrane and secreted growth cone guidance molecules. Cell 75: 1389-1399, 1993.

[14] Yazdani U and Terman JR: The semaphorins. Genome Biol 7: 211, 2006.

[15] Ito D, Nojima S and Kumanogoh A: The role of semaphorin family in immune systems. Nihon Rinsho Meneki Gakkai Kaishi 37: 1-10, 2014.

[16] Kong-Beltran M, Stamos J and Wickramasinghe D: The Sema domain of Met is necessary for receptor dimerization and activation. Cancer Cell 6: 75-84, 2004.

[17] Jin MS, Park IA, Kim JY, Chung YR, Im SA, Lee KH, Moon HG, Han W, Noh DY and Ryu HS: New insight on the biological role of p53 protein as a tumor suppressor: re-evaluation of its clinical significance in triple-negative breast cancer. Tumour Biol 37: 11017-11024, 2016.

[18] Moriya J, Minamino T, Tateno K, Okada S, Uemura A, Shimizu I, Yokoyama M, Nojima A, Okada M, Koga H and Komuro I: Inhibition of semaphorin as a novel strategy for therapeutic angiogenesis. Circ Res 106: 391-398, 2010.

[19] Chen R, Zhuge X, Huang Z, Lu D, Ye X, Chen C, Yu J and Lu G: Analysis of SEMA3B methylation and expression patterns in gastric cancer tissue and cell lines. Oncol Rep 31: 1211-1218, 2014.

[20] Ito M, Ito G, Kondo M, Uchiyama M, Fukui T, Mori S, Yoshioka H, Ueda Y, Shimokata K and Sekido Y: Frequent inactivation of RASSF1A, BLU, and SEMA3B on 3p21.3 by promoter hypermethylation and allele loss in non-small cell lung cancer. Cancer Lett 225: 131-139, 2005.

[21] Zhang L, Wang JN, Tang JM, Kong X, Yang JY, Zheng F, Guo LY, Huang YZ, Zhang L, et al: VEGF is essential for the growth and migration of human hepatocellular carcinoma cells. Mol Biol Rep 39: 5085-5093, 2012. 
[22] Varshavsky A, Kessler O, Abramovitch S, Kigel B, Zaffryar S, Akiri G and Neufeld G: Semaphorin-3B is an angiogenesis inhibitor that is inactivated by furin-like pro-protein convertases. Cancer Res 68: 6922-6931, 2008.

[23] Jauhiainen S, Hakkinen SK, Toivanen PI, Heinonen SE, Jyrkkanen HK, Kansanen E, Leinonen H, Levonen AL and Yla-Herttuala S: Vascular endothelial growth factor (VEGF)-D stimulates VEGF-A, stanniocalcin-1, and neuropilin-2 and has potent angiogenic effects. Arterioscler Thromb Vasc Biol 31: 1617-1624, 2011.

[24] Tomizawa Y, Sekido Y, Kondo M, Gao B, Yokota J, Roche J, Drabkin H, Lerman MI, Gazdar AF and Minna JD: Inhibition of lung cancer cell growth and induction of apoptosis after reexpression of $3 \mathrm{p} 21.3$ candidate tumor suppressor gene SEMA3B. Proc Natl Acad Sci U S A 98: 13954-13959, 2001.

[25] de Lange R, Dimoudis N and Weidle UH: Identification of genes associated with enhanced metastasis of a large cell lung carcinoma cell line. Anticancer Res 23: 187-194, 2003.

[26] Beuten J, Garcia D, Brand TC, He X, Balic I, Canby-Hagino E, Troyer DA, Baillargeon J, Hernandez J, et al: Semaphorin 3B and $3 \mathrm{~F}$ single nucleotide polymorphisms are associated with prostate cancer risk and poor prognosis. J Urol 182: 1614-1620, 2009.

[27] Karayan-Tapon L, Wager M, Guilhot J, Levillain P, Marquant C, Clarhaut J, Potiron V and Roche J: Semaphorin, neuropilin and VEGF expression in glial tumours: SEMA3G, a prognostic marker? Br J Cancer 99: 1153-1160, 2008.

[28] Chen HC, Huang HY, Chen YL, Lee KD, Chu YR, Lin PY, Hsu CC, Chu PY, Huang TH, et al: Methylation of the Tumor Suppressor Genes HIC1 and RassF1A Clusters Independently From the Methylation of Polycomb Target Genes in Colon Cancer. Ann Surg Oncol 2015.

[29] Sheng Y, Wang H, Liu D, Zhang C, Deng Y, Yang F, Zhang T and Zhang C: Methylation of tumor suppressor gene CDH13 and SHP1 promoters and their epigenetic regulation by the UHRF1/PRMT5 complex in endometrial carcinoma. Gynecol Oncol 140: 145-151, 2016.
[30] Xie M, Wu X, He C, Zhang J and Zhang J: Ski is silenced by methylation and acts as tumor suppressor in non-small cell lung cancer. Oncotarget 2015.

[31] Gao X, Tang C, Shi W, Feng S, Qin W, Jiang T and Sun Y: Semaphorin-3F functions as a tumor suppressor in colorectal cancer due to regulation by DNA methylation. Int J Clin Exp Pathol 8: 12766-12774, 2015.

[32] Kuroki T, Trapasso F, Yendamuri S, Matsuyama A, Alder H, Williams NN, Kaiser LR and Croce CM: Allelic loss on chromosome $3 \mathrm{p} 21.3$ and promoter hypermethylation of semaphorin 3B in non-small cell lung cancer. Cancer Res 63: 3352-3355, 2003.

[33] Nair PN, McArdle L, Cornell J, Cohn SL and Stallings RL: High-resolution analysis of $3 p$ deletion in neuroblastoma and differential methylation of the SEMA3B tumor suppressor gene. Cancer Genet Cytogenet 174: 100-110, 2007.

[34] Yi HM, Li H, Peng D, Zhang HJ, Wang L, Zhao M, Yao KT and Ren CP: Genetic and epigenetic alterations of LTF at $3 \mathrm{p} 21.3$ in nasopharyngeal carcinoma. Oncol Res16: 261-272, 2006.

[35] Riquelme E, Tang M, Baez S, Diaz A, Pruyas M, Wistuba, II and Corvalan A: Frequent epigenetic inactivation of chromosome $3 \mathrm{p}$ candidate tumor suppressor genes in gallbladder carcinoma. Cancer Lett 250: 100-106, 2007.

[36] Osada R, Horiuchi A, Kikuchi N, Ohira S, Ota M, Katsuyama $\mathrm{Y}$ and Konishi I: Expression of semaphorins, vascular endothelial growth factor, and their common receptor neuropilins and alleic loss of semaphorin locus in epithelial ovarian neoplasms: increased ratio of vascular endothelial growth factor to semaphorin is a poor prognostic factor in ovarian carcinomas. Hum Pathol 37: 1414-1425, 2006.

[37] Rolny C, Capparuccia L, Casazza A, Mazzone M, Vallario A, Cignetti A, Medico E, Carmeliet P, Comoglio PM and Tamagnone L: The tumor suppressor semaphorin 3B triggers a prometastatic program mediated by interleukin 8 and the tumor microenvironment. J Exp Med 205: 1155-1171, 2008. 